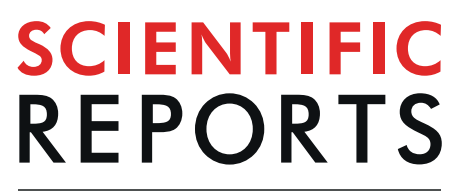

natureresearch

\title{
Gene expression profile analysis of ileum transcriptomes in pigs fed Gelsemium elegans plants
}

\begin{abstract}
Chong-Yin Huang ${ }^{1,2}$, Kun Yang ${ }^{1,2}$, Jun-Jie Cao ${ }^{1,2}$, Yu-Juan Li, ${ }^{1,2}$, Zi-Yuan Wang ${ }^{1,2}$, Hui Yu ${ }^{1,2}$, ZhiLiang Sun ${ }^{1,2}$, Xiaofeng Zheng ${ }^{1,2}$ \& Zhao-Ying Liu $\mathbb{1}^{1,2^{*}}$

Gelsemium elegans is a flowering plant in the Loganiaceae. Because it can promote the growth of pigs and sheep, it is widely used, including in veterinary clinics, but little information is available about its biological effects. Here, we used high-throughput sequencing to characterize the differentially expressed genes (DEGs) in the ileums of pigs between a control group and a group fed Gelsemium elegans for 45 days. We found that Gelsemium elegans affected many inflammatory and immune pathways, including biological processes such as defense responses, inflammation and immune responses. Moreover, this study identified several important genes related to the anti-inflammatory activity of Gelsemium elegans (e.g., CXCL-8, IL1A, and CSF2), which will be beneficial for further study of the pharmacological mechanisms and clinical applications of Gelsemium elegans.
\end{abstract}

Gelsemium is an herb in the family Loganiaceae. Gelsemium mainly includes three species with different regional distributions: Gelsemium sempervirens (L.), Gelsemium rankinii Small, and Gelsemium elegans Benth. Gelsemium elegans is mainly distributed in southeast Asia and has been used as a traditional Chinese medicine for treating neuropathic pain, rheumatoid pain, spasms, skin ulcers and cancer for many years ${ }^{1,2}$. In China, the plant is mainly distributed near the middle and lower reaches of the Yangtze River, especially in the warm coastal areas of the Fujian and Guangxi provinces. However, Gelsemium elegans, a poisonous plant, exerts obvious toxicity toward the human body. Poisoning has often been reported after people have eaten Gelsemium elegans by mistake ${ }^{3}$. After poisoning, patients suffer from symptoms such as dizziness, sweating, nausea, vomiting, muscle relaxation, limb paralysis, dyspnea, coma and convulsion ${ }^{4,5}$. The nervous system is depressed, and death is caused by respiratory depression $^{4-6}$; This toxicity limits the applications of this plant.

Interestingly, in Chinese folk medicine, Gelsemium elegans is often used in animals to prevent parasite infestation and to promote growth. Its effect is obvious in pigs and sheep. In the Compendium of Materia Medica, it is recorded that people who eat Gelsemium by mistake die, while sheep that eat Gelsemium grow large and fat ${ }^{7}$. Chen et al. recorded that Gelsemium, which is regularly given to pigs and sheep, can invigorate the stomach, kill parasites and fatten the animals ${ }^{8}$. These statements show that Gelsemium not only is nontoxic when eaten by animals such as pigs and sheep but also has the effects of fattening the animals and preventing disease. Therefore, it is critical to study the mechanisms of the effects of Gelsemium elegans on the pig intestinal tract.

In this experiment, high-throughput transcriptome sequencing technology was used to examine changes at the transcriptome level in the pig ileum under the influence of Gelsemium elegans. High-throughput sequencing has characteristics including a short sequencing time, low cost, and high sequence quantity ${ }^{9}$. Transcriptomics, an important component of functional genomics research, can be used to study overall gene function and structure, revealing specific biological processes and molecular mechanisms that are involved in the process of disease occurrence ${ }^{10}$. Therefore, the purposes of this study were to clarify the possible mechanisms of the effects of Gelsemium elegans on the intestinal tract as a whole based on high-throughput sequencing, to provide new data to reveal the molecular mechanisms of the pharmacological effects of Gelsemium elegans and to provide important references for further in-depth study of the mechanisms of the effects of Gelsemium elegans.

\footnotetext{
${ }^{1}$ Hunan Engineering Technology Research Center of Veterinary Drugs, Hunan Agricultural University, Changsha, 410128, China. ${ }^{2}$ College of Veterinary Medicine, Hunan Agricultural University, Changsha, 410128, China. *email: liv_zhaoying@hunau.edu.cn
} 


\begin{tabular}{|c|c|c|c|c|c|c|}
\hline Sample_name & $\mathrm{Cl}$ & $\mathrm{C} 2$ & C3 & G1 & G2 & G3 \\
\hline Raw reads & 53351960 & 52203452 & 62621280 & 50860180 & 53702840 & 40267710 \\
\hline Clean reads & 52354682 & 50836840 & 61569634 & 49898956 & 52727580 & 39636068 \\
\hline Clean bases $(\mathrm{Gb})$ & 7.39 & 7.24 & 8.70 & 7.05 & 7.49 & 5.63 \\
\hline Q20(\%) & $97.10 \%$ & $96.95 \%$ & $97.10 \%$ & $96.80 \%$ & $96.95 \%$ & $97.45 \%$ \\
\hline Q30(\%) & $92.20 \%$ & $91.90 \%$ & $92.20 \%$ & $91.60 \%$ & $91.80 \%$ & $92.95 \%$ \\
\hline Total mapped & $50591699(96.60 \%)$ & $49185332(96.80 \%)$ & $58949697(95.70 \%)$ & $47391403(95 \%)$ & $50409079(95.60 \%)$ & $38280432(96.60 \%)$ \\
\hline Multiple mapped & 2682859 & 2066081 & 3841841 & 3278765 & 3483805 & 2173146 \\
\hline Uniquely mapped & 47908840 & 47119251 & 55107856 & 44112638 & 46925274 & 36107286 \\
\hline Reads map to '+' & 23954389 & 23559590 & 27553907 & 22056319 & 23462604 & 18053653 \\
\hline Reads map to '-‘ & 23954451 & 23559661 & 27553949 & 22056319 & 23462670 & 18053633 \\
\hline Non-Splice reads & 26179110 & 26211625 & 31002946 & 24250115 & 26227333 & 20306368 \\
\hline Splice reads & 21729730 & 20907626 & 24104910 & 19862523 & 20697941 & 15800918 \\
\hline
\end{tabular}

Table 1. Statistics from digital gene expression sequencing. C1 3: ileum tissue samples from the control group; G1 3: ileum tissue samples from the experimental group.

\begin{tabular}{|c|c|c|c|c|c|c|}
\hline \multirow[b]{2}{*}{ Group } & \multicolumn{6}{|l|}{ FPKM interval } \\
\hline & 0 1 & $1 \sim 5$ & $5 \sim 10$ & $10 \sim 20$ & $20 \sim 50$ & $>50$ \\
\hline $\mathrm{C} 1$ & $7510(37.81 \%)$ & $4396(22.13 \%)$ & $2955(14.88 \%)$ & $2350(11.83 \%)$ & $1690(8.51 \%)$ & $961(4.84 \%)$ \\
\hline $\mathrm{C} 2$ & 7754 (39.04\%) & $4578(23.05 \%)$ & $2760(13.9 \%)$ & $2268(11.42 \%)$ & $1525(7.68 \%)$ & $977(4.92 \%)$ \\
\hline $\mathrm{C} 3$ & $8249(41.53 \%)$ & $4632(23.32 \%)$ & $2622(13.2 \%)$ & $2040(10.27 \%)$ & $1403(7.07 \%)$ & $916(4.61 \%)$ \\
\hline G1 & $8087(40.72 \%)$ & $4722(23.77 \%)$ & $2785(14.02 \%)$ & $2066(10.4 \%)$ & $1360(6.85 \%)$ & $842(4.24 \%)$ \\
\hline G2 & $8035(40.45 \%)$ & $4641(23.37 \%)$ & $2718(13.68 \%)$ & $2156(10.85 \%)$ & $1416(7.12 \%)$ & $896(4.51 \%)$ \\
\hline G3 & $7602(38.27 \%)$ & $4620(23.26 \%)$ & $2905(14.63 \%)$ & $2380(11.98 \%)$ & $1467(7.39 \%)$ & $888(4.47 \%)$ \\
\hline
\end{tabular}

Table 2. The number of genes in different FPKM expression intervals in all samples.

\section{Results}

Transcriptome sequencing data quality testing. Transcriptome sequencing was performed on a total of 6 samples from the experimental group and the control group (Table 1), and an average of 52.168 M reads were obtained. After removing the linker fragments and the low-quality fragments, a total of 51.171 $\mathrm{M}$ high-quality clean reads were obtained; the Q20\% of the clean reads in each sample was above $96.80 \%$, and the Q30\% was above $91.60 \%$, indicating that the sequencing quality was good and provided good original data for subsequent data assembly. Upon comparing the clean reads to the reference genome, an average of $49.135 \mathrm{M}$ could be mapped, which accounted for $96 \%$ of the total number of sequences. The comparison showed that the alignment efficiency was good.

Gene expression statistics. HTSeq ${ }^{11}$ was used to quantify the expression level of each sample gene to obtain the raw counts, which were the input data for the next differential expression analysis. Then, the raw counts for each sample were standardized to obtain the FPKM value of each sample gene, and the expression levels of six sample genes were determined (Table 2 ). The number of highly expressed genes (FPKM $>50)$ in each ileum sample was maintained at approximately 800 , while the number of genes with low expression or no expression $(\mathrm{FPKM}<1)$ was above 7000 in all samples.

Screening of differentially expressed genes. Taking a $\mid \log 2$ (fold change) $\mid \geq 1$, a P-value $\leq 0.05$ and an FDR (adjusted for P-value) $\leq 0.05$ as the screening conditions, the experimental group and the control group were compared and analyzed. A total of 446 differentially expressed genes (DEGs) were screened in this experiment, of which 237 genes were upregulated and 209 genes were downregulated.

GO classification of differentially expressed genes. GO annotation was conducted for the DEGs, and a total of 30 significant GO terms were enriched. As shown in Fig. 1, the DEGs were mainly enriched in biological processes such as defense responses, humoral immune responses, inflammatory responses, and immune system processes, which indicates that intake of Gelsemium elegans leads to changes in immune and inflammatory response processes in animal bodies. In the cellular component category, the cell periphery, plasma membrane, extracellular region and plasma membrane part terms were most prominent; in the molecular function category, receptor activity/receptor binding was the main term.

Enrichment of differentially expressed gene pathways. As shown in Fig. 2, 446 DEGs between the experimental group and the control group were enriched in signaling pathways related to inflammation and the immune response, including the complement and coagulation cascade pathway, the IL-17 signaling pathway, the rheumatoid arthritis pathway and the hematopoietic cell lineage pathway, with relatively high enrichment rates. 


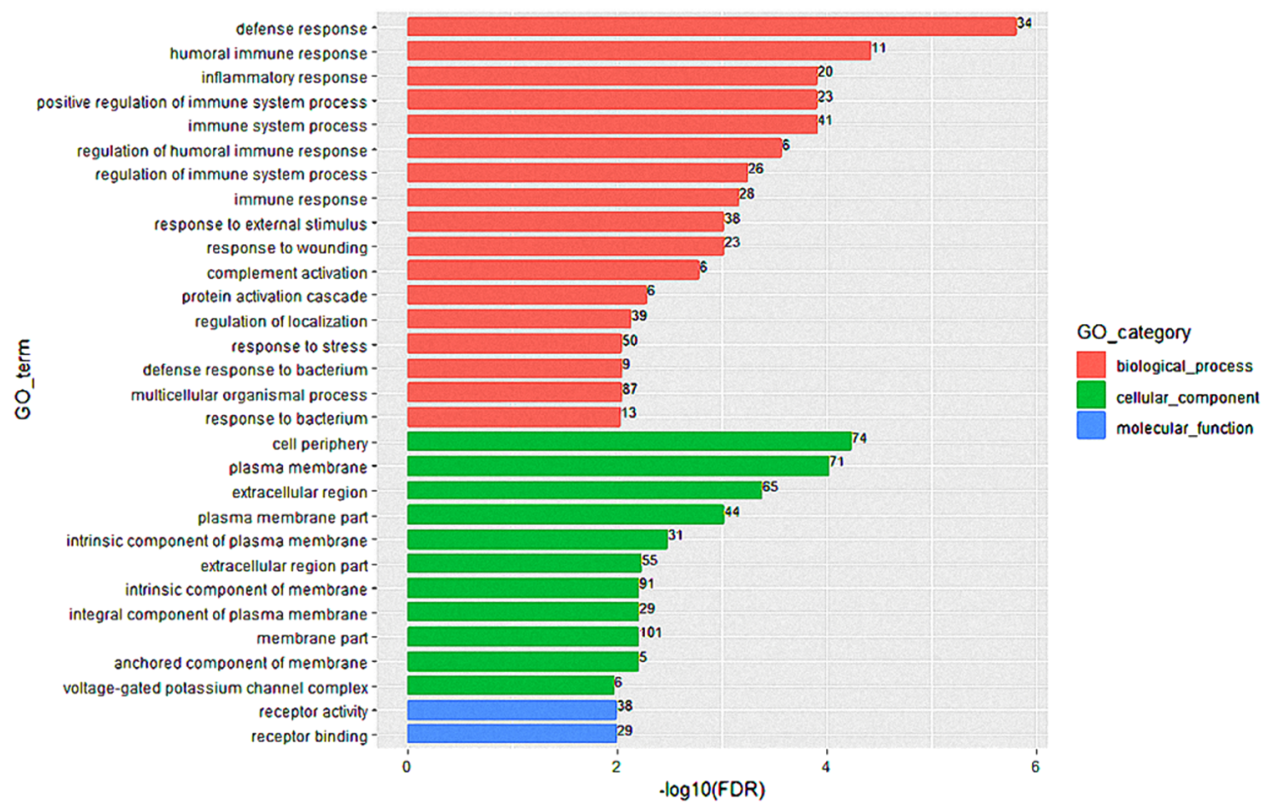

Figure 1. GO classifications of the differentially expressed genes in intestinal tissues from the Gelsemium elegans group and the control group. The differentially expressed genes were classified into three categories: biological process, cellular component, and molecular function.

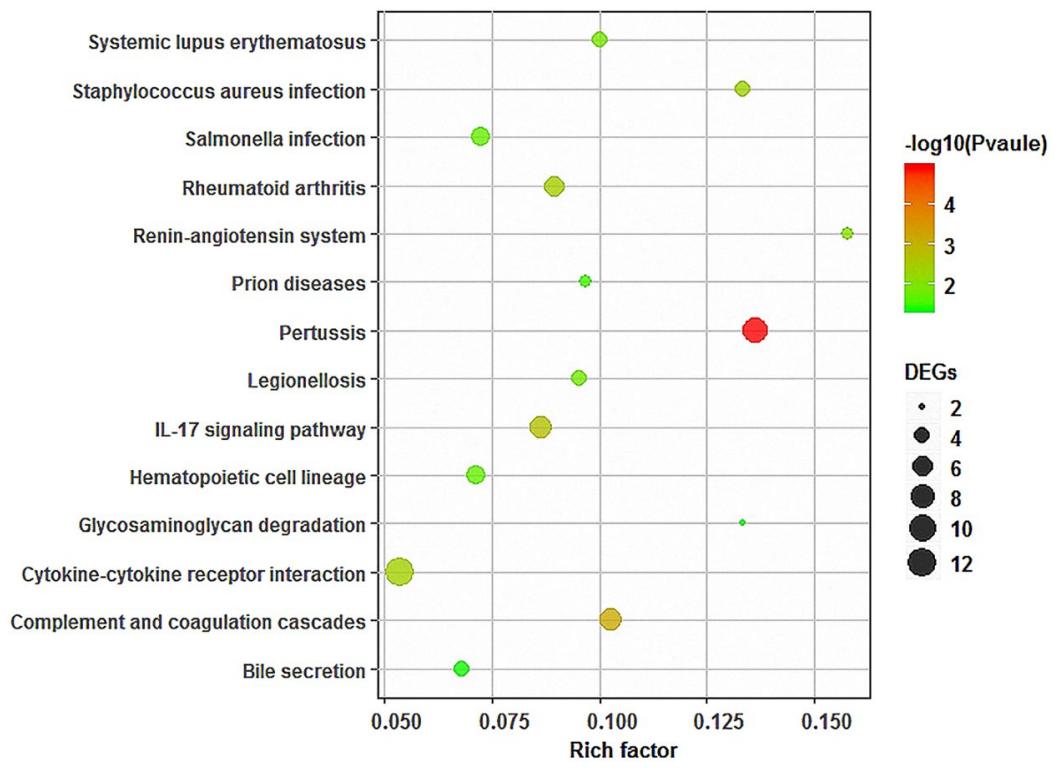

Figure 2. Pathway enrichment results of the differentially expressed genes. The X-axis represents the enrichment factor value, and the Y-axis represents the pathway name. The color represents the P-value (significance: $\mathrm{P} \leq 0.05$ ), and the size of the point represents the number of genes. The enrichment factor represents the ratio of the enriched genes to the background genes.

The genes enriched in the pathways are shown in Table 3. Statistical analyses showed that CXCL-8, IL1A, C3, C5, CSF2, C2, CXCL-2, FOS, AMCF-II, and C8G were involved in at least three of the above pathways, suggesting that these genes may be important hubs for communication between different signaling pathways. Further statistical analyses showed that CXCL-8, CSF2 and AMCF-II are all involved in the cytokine-cytokine receptor interaction pathway and the rheumatoid arthritis pathway, which are related to inflammation, suggesting that these two pathways may be key pathways in inflammation-related reaction processes.

QRT-PCR verification results. The correlation analysis results of the qRT-PCR and RNA-seq data were similar (Fig. 3), which suggests that the transcriptome sequencing data are highly reproducible and accurate. 


\begin{tabular}{|c|c|c|c|c|c|}
\hline Gene symbol & Gene description & Gene ID & Gene family & \begin{tabular}{|l|}
$\log _{2}($ Fold \\
Change)
\end{tabular} & Pvalue \\
\hline IL1RL2 & interleukin 1 receptor like 2 & ENSSSCG00000023550 & - & 3.55 & 0.0002 \\
\hline IL5RA & interleukin 5 receptor subunit alpha & ENSSSCG00000011528 & chemokine receptor family & -1.36 & 0.0191 \\
\hline CCL28 & C-C motif chemokine ligand 28 & ENSSSCG00000016871 & Intercrine beta(chemokine CC)family & -1.37 & $<0.0001$ \\
\hline CCR2 & C-C chemokine receptor type 2 & ENSSSCG00000024311 & - & -1.16 & $<0.0001$ \\
\hline LTA & Sus scrofa lymphotoxin alpha (LTA), mRNA. & ENSSSCG00000001403 & tumor necrosis factor family. & 2.61 & 0.0289 \\
\hline CSF2 & granulocyte-macrophage colony-stimulating factor precursor & ENSSSCG00000023737 & GM-CSF family & -2.02 & 0.0003 \\
\hline FOS & proto-oncogene c-Fos & ENSSSCG00000002383 & FOS subfamily & 1.06 & $<0.0001$ \\
\hline CXCL8 & Sus scrofa C-X-C motif chemokine ligand 8 (CXCL8), mRNA. & ENSSSCG00000008953 & CXC chemokine family & -1.21 & 0.0007 \\
\hline CXCL2 & C-X-C motif chemokine 2 precursor & ENSSSCG00000008959 & CXC chemokine family & -1.73 & 0.0011 \\
\hline S100A8 & Sus scrofa S100 calcium binding protein A8 (S100A8), mRNA. & ENSSSCG00000006590 & S-100 family & -3.05 & 0.0074 \\
\hline ANPEP & alanyl aminopeptidase, membrane & ENSSSCG00000001849 & peptidase M1 family & 1.01 & 0.0113 \\
\hline CD38 & ADP-ribosyl cyclase/cyclic ADP-ribose hydrolase 1 & ENSSSCG00000008742 & ADP-ribosyl cyclase family & -1.03 & $<0.0001$ \\
\hline С4BPB & complement component 4 binding protein beta & ENSSSCG00000015661 & - & -1.64 & $<0.0001$ \\
\hline С4BPA & $\begin{array}{l}\text { Sus scrofa complement component } 4 \text { binding protein, alpha } \\
\text { (C4BPA), mRNA. }\end{array}$ & ENSSSCG00000015662 & - & -1.18 & 0.0110 \\
\hline C8G & complement $\mathrm{C} 8$ gamma chain & ENSSSCG00000005840 & calycin superfamily & 2.17 & 0.0217 \\
\hline F11 & - & ENSSSCG00000032653 & peptidase S1 family & -1.71 & 0.0241 \\
\hline $\mathrm{C} 2$ & complement $\mathrm{C} 2$ & ENSSSCG00000001422 & peptidase S1 family & -1.01 & $<0.0001$ \\
\hline C3 & complement C3 & ENSSSCG00000013551 & - & -1.52 & 0.0006 \\
\hline C5 & complement C5 & ENSSSCG00000005512 & - & -1.26 & 0.0010 \\
\hline
\end{tabular}

Table 3. Pathway enrichment gene expression.

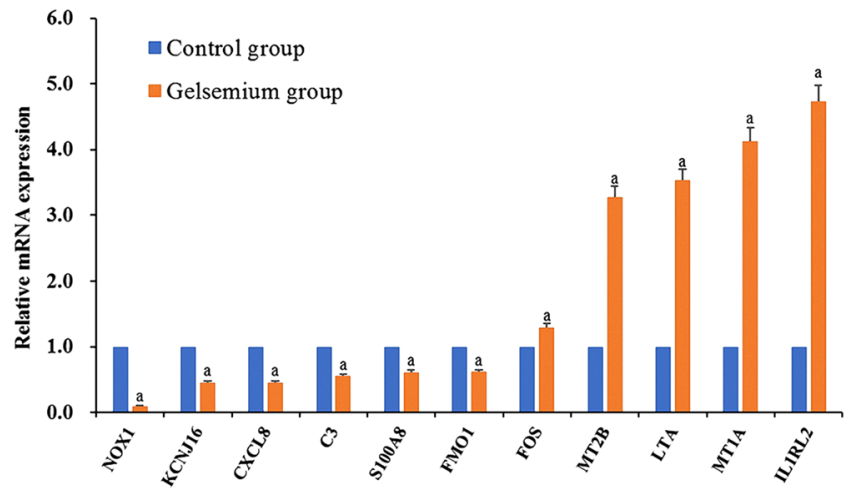

Figure 3. Relative expression of the candidate genes by qRT-PCR. Three samples were included in each group, and three replicates for each sample were performed. The relative expression level was measured by qRT-PCR. These data are expressed as the mean $\pm \mathrm{SD}$ relative to the control. ${ }^{\mathrm{a}} \mathrm{P}<0.05$ compared with the control group.

\section{Discussion}

RNA-seq technology is an ideal high-throughput sequencing method that greatly improves the speed and efficiency of identifying new genes or biomarkers ${ }^{12,13}$. Therefore, in this study, the RNA-seq technique was used to determine the transcriptomic effects of Gelsemium elegans on the pig ileum, and 446 DEGs were obtained. A total of 237 genes were upregulated, and 209 genes were downregulated in the ileum tissue of the experimental group compared with that of the control group. At the same time, we found 14 important pathways. These results provide the basis for a better understanding of the molecular mechanisms of Gelsemium elegans in pigs.

The results of expression analysis of inflammatory response-related genes showed that the inflammatory genes in the experimental group were generally downregulated. First, genes associated with inflammatory-related 
receptors and signaling pathways, such as the cytokine-cytokine receptor interaction pathway and the IL-17 signaling pathway, suggesting that cytokine-cytokine receptor action is inhibited and that intercellular signal transmission is weakened under Gelsemium treatment ${ }^{14,15}$. Most of the genes enriched in inflammatory response-related pathways, such as the rheumatoid arthritis pathway, and related metabolic processes in inflammation, such as the complement and coagulation cascade pathways, were downregulated, indicating that the intestinal inflammatory response of the experimental group was inhibited.

The downregulated genes associated with the complement and coagulation cascades pathway in the experimental group compared with the control group were C4BPB, C4BPA, F11, C2, C3 and C5, among which C3 is a local inflammatory reaction process mediator that can induce smooth muscle contraction, increase vascular permeability and promote mast cells and leukocytes to release histamine. C3 also plays the role of an inflammatory cell chemical inducer in chronic inflammation. In addition, C5 is an effective chemokine that stimulates leukocyte movement and induces leukocytes to migrate to inflammatory sites. The complement and coagulation cascades were all downregulated, which may have been related to the inhibition of inflammatory reactions. This finding shows that Gelsemium elegans might reduce inflammatory cell infiltration by reducing the levels of cytokines related to the complement and coagulation cascades.

The IL-17 signaling pathway is involved in the pathogenesis of many inflammatory and autoimmune diseases, including rheumatoid arthritis ${ }^{16}$ and psoriasis. S100A8 is a calcium-zinc binding protein (with significantly downregulated expression) belonging to the low-molecular weight S100 protein family that participates in the migration process of neutrophils to inflammatory sites $^{1}$, can bind with TLR 4 and AGER receptors, and activates the MAP kinase and NF-K-B signaling processes, thus leading to expansion of the proinflammatory cascade and playing an important role in inflammatory process and immune response regulation.

In the rheumatoid arthritis pathway, a total of 5 DEGs were enriched, among which IL-8/CXCL-8 is a key mediator related to inflammation belonging to the CXC family of chemokines that is usually released in response to inflammatory stimulation. IL-8/CXCL-8 plays a key role in neutrophil recruitment, and degranulation ${ }^{17}$ is considered to be an inflammatory mediator of gingivitis ${ }^{18}$ and psoriasis. In a study of Gelsemium elegans, some researchers developed an injectable solution and oral liquid from a water extract of Gelsemium elegans and applied it to the treatment of psoriasis and neurodermatitis ${ }^{19}$. The downregulation of the CXCL- 8 gene is consistent with the anti-inflammatory activities of Gelsemium elegans and emphasizes the role of CXCL-8 in the pharmacological activities of Gelsemium elegans. CSF2, also known as granulocyte-macrophage colony-stimulating factor (GM-CSF), is a monomeric glycoprotein. Clinical tests have revealed its proinflammatory effect. Studies have shown that CSF2 is highly expressed in joints with rheumatoid arthritis, and inflammation and injury can be reduced by blocking CSF2. IL-1 $\alpha / \mathrm{IL} 1 \mathrm{~A}$, which are members of the IL- 1 cytokine family together with IL-1 $\beta$, can induce the release of IL-2 and mainly play a role in regulating and initiating inflammatory reactions. Studies have found that blocking the activity of IL-1 $\alpha$ is likely to be useful for the treatment of skin diseases such as acne ${ }^{20}$. IL-1 $\beta$ is an important mediator of inflammatory reactions that can induce many kinds of self-inflammatory diseases. Experiments have found that intestinal ecological imbalance can induce osteomyelitis in an IL-1 $\beta$-dependent manner ${ }^{21}$.

A large number of studies have indicated that Gelsemium elegans has strong anti-inflammatory activity ${ }^{22-24}$. Researchers have used transcriptome analysis to study SH-SY5Y neurons in humans treated with low concentrations of Gelsemium alkaloids and found significant changes in related regulatory genes of the inflammatory response signaling pathway ${ }^{23}$. Some researchers have also found that medium and high doses of Gelsemium could significantly inhibit the mitosis process in vaginal epithelial cells and promote the formation of epidermal granules in mouse tail scales. At the same time, the level of IL-2 in the serum of experimental mice was found to be significantly reduced in three dose groups ${ }^{24}$. Koumine, which is one of the major Gelsemium alkaloids, has been found to reduce the levels of the proinflammatory factors TNF and IL-1, attenuate collagen-induced increases in fibrillary protein content, and inhibit astrocyte activation and proinflammatory factor-mediated pain ${ }^{25,26}$.

Using qPCR and Western blot analysis, Yuan et al. found that koumine can inhibit the release of inflammatory mediators and cytokines such as TNF- $\alpha$, IL- 6 and IL- $1 \beta^{27}$. Similarly, studies have shown that IL- $1 \alpha^{28}$, CXCL $8^{29}$, $\mathrm{CSF} 2^{30}, \mathrm{C}^{31}, \mathrm{C}^{32}$ and S100A $8^{33}$ were consistent at the mRNA and protein levels. However, Yang et al. found that koumine can selectively inhibit the proliferation of T cells, especially CD4+ T (Th) cells, reducing the levels of inflammatory cytokines (Th 1 cytokines IFN- $\gamma$, IL-1 $\beta, 2,6,12$, TNF- $\alpha$ and Th17- $\alpha$ cytokines IL-17A) ${ }^{34}$. Aggarwal A. et al. found that rheumatoid arthritis is closely related to polarity deviation in Th cells and systemic disorder of inflammatory and anti-inflammatory cell networks ${ }^{35}$. It is worth noting that macrophages can release IL-1 $\alpha$, which acts on Th cells to regulate their activity. These studies suggest that Gelsemium elegans may regulate the polarity deviation of Th cells by inhibiting the expression of IL- $1 \alpha$, thus reducing the production of inflammatory factors. In this study, the expression of many inflammatory cytokine genes was inhibited, and the levels of these cytokines entering the blood to reach corresponding lesions were decreased; these changes achieved an anti-inflammatory effect by restoring the balance of the network.

\section{Conclusions}

In this study, we reported the ileum transcriptome profiles of three-way hybrid weaned piglets fed two different diets (a complete diet without antibacterial drugs and a complete diet containing $2 \%$ Gelsemium elegans herb without antibacterial drugs) and carried out detection and DEG identification. The results showed that Gelsemium elegans affected many inflammatory and immune pathways, including biological processes such as defense responses, inflammation and immune responses. More importantly, this study identified several important genes (e.g., CXCL-8, IL1A, and CSF2). These data provide a clearer understanding for elucidating the molecular mechanisms of the pharmacological actions of Gelsemium, such as its anti-inflammatory effects. 


\begin{tabular}{|l|l|}
\hline Ingredients & Ratio (\%) \\
\hline Corn & 52.99 \\
\hline Expanded corn & 10.00 \\
\hline Soybean meal & 20.00 \\
\hline Fish meal & 1.50 \\
\hline Soybean oil & 0.80 \\
\hline Fine powder & 0.72 \\
\hline Salt & 0.30 \\
\hline Sucrose & 2.50 \\
\hline Premix ${ }^{\mathrm{a}}$ & 2.59 \\
\hline Total & 100.00 \\
\hline Nutrient levels ${ }^{\mathbf{b}}$ & \multicolumn{2}{|l}{} \\
\hline DE (MJ/kg) & 14.20 \\
\hline Crude Protein(\%) & 19.00 \\
\hline Calcium (\%) & 0.80 \\
\hline Available phosphorus (\%) & 0.50 \\
\hline Lysine (\%) & 1.40 \\
\hline Methionine (\%) & 0.84 \\
\hline L-Threonine (\%) & 0.40 \\
\hline & \multicolumn{1}{|l}{} \\
\hline
\end{tabular}

Table 4. Composition and nutritional level of the complete diet. ${ }^{(a)}$ Premix is provided per kilogram of daily ration: VA $2200 \mathrm{IU}$; VD $220 \mathrm{IU}$; VE $62 \mathrm{mg}$; VK3 $3.00 \mathrm{mg}$; VB1 $3.00 \mathrm{mg}$; VB6 $3.60 \mathrm{mg}$; VB12 $0.02 \mathrm{mg}$; Biotin $0.45 \mathrm{mg}$; Pantothenic acid $10 \mathrm{mg}$; Nicotinic acid $30 \mathrm{mg}$; Choline chloride $500 \mathrm{mg}$; $\mathrm{Mn} 50 \mathrm{mg}$; Fe $150 \mathrm{mg}$; $\mathrm{Zn}$ $2200 \mathrm{mg}$; Cu $200 \mathrm{mg}$; I $0.3 \mathrm{mg}$; Se $0.3 \mathrm{mg}$. ${ }^{(b)}$ The crude protein level in the table is the measured value, and the other is the calculated value.

\begin{abstract}
Materials and Methods
Animals and treatments. In this study, 6 healthy castrated male ternary hybrid piglets approximately 60 days old and weighing $20 \pm 2 \mathrm{~kg}$ were obtained from Hunan Xinwufeng, Yong'an branch (Hunan, China), and randomly divided into control and Gelsemium elegans treatment groups. The control group was a blank control group fed a complete diet without antibacterial drugs, and the Gelsemium elegans group was an experimental group. The diet was prepared by adding $2 \%$ Gelsemium elegans powder to a complete diet without antibacterial drugs. The formula and nutritional level of the complete diet are shown in Table 4 . After 45 days of continuous feeding, administration was stopped, and all piglets were fasted for 24 hours. The piglets were slaughtered within one day after stopping the drug, and a sterile blade was used to take ileum tissue from the control group and the experimental group. The tissue samples were washed in distilled water to remove blood and placed into centrifuge tubes. The tubes were sealed with sealing film and quickly placed in liquid nitrogen for storage. The samples were stored at $-80^{\circ} \mathrm{C}$ until RNA extraction. This experiment was carried out in accordance with the Guidelines for the Care and Use of Laboratory Animals of China and approved by the Animal Care and Use Committee of the Institute of Subtropical Agriculture, Chinese Academy of Sciences (IACUC\# 201302).
\end{abstract}

Extraction of total RNA. Total RNA was isolated from each sample using TRIzol reagent (Invitrogen, Carlsbad, CA, USA) according to the manufacturer's protocol. The integrity and purity of the RNA were detected by agarose gel electrophoresis (Tianneng, China) and with a NanoDrop (Thermo, USA), and the RNA concentration was accurately quantified with a Qubit instrument (Invitrogen, USA).

Construction of the sequencing library. The mRNA was enriched by magnetic beads with Oligo(dT), and then, the mRNA was randomly broken into short fragments of approximately $200 \mathrm{bp}$. Using mRNA as a template, first-strand cDNA was synthesized by reverse transcription using random primers. For synthesis of the second strand of cDNA, dTTP in the dNTP mixture was replaced by dUTP. The CDNA product was purified with AMPure XP beads (Beckman, USA); then, the double-stranded ends were blunted with End Repair Mix, and poly-A tails and sequencing linkers were added. The USER enzyme was used to digest the double-stranded cDNA to make a library containing only single-stranded cDNA. PCR (Life, USA) enrichment was performed, and the final cDNA library was obtained after 15 cycles of amplification.

Transcriptome sequencing. An Illumina HiSeq sequencing platform (Illumina, USA) was used, and the $2 * 150$ sequencing mode was adopted to construct a qualified cDNA library for double-terminal (PE) sequencing by Shanghai Jingneng Biotechnology Limited (Shanghai, China).

Processing and analysis of sequencing results. According to the distribution feature that the low-mass fraction of Illumina sequencing data is usually concentrated at the end, the raw sequencing data were filtered to obtain clean reads. Trim Galore (http://www.bioinformatics.babraham.ac.uk/projects/trim_galore/) software was used to remove the linker sequence fragments and low-mass fragments from the 3' ends of the raw data. Finally, high-quality clean reads were obtained, and the clean reads and pig reference genome sequences were 
sequence-matched with STAR software ${ }^{36}$. The obtained alignment results were based on the positional information files of the known transcripts on the genome, and transcripts with expression values of 0 were removed with StringTie software ${ }^{37}$. Then, the transcripts for all samples assembled with StringTie were reassembled to obtain new transcripts. BLASTX software was used to carry out sequence alignment with the UniProt (http://www. uniprot.org/downloads), GO (http://geneontology.org/) and KEGG (http://www.genome.jp/) databases to obtain corresponding unigene functional annotation information.

Differential expression analysis. The FPKM values were used to calculate the gene expression levels in the samples, and differential expression was screened between the experimental group samples and the control group samples using DESeq. 2 software. DEGs were identified by using a $\log 2 \mid$ fold change $\mid \geq 1$, a P-value $\leq 0.05$ and an FDR (adjusted for P-value) $\leq 0.05$ as the screening conditions. GO functional enrichment analysis ${ }^{38}$ and KEGG pathway enrichment analysis ${ }^{39}$ for the DEGs were performed.

Validation of RNA-seq data by quantitative real-time PCR (qRT-PCR). Validation was performed by using the same RNA used for sequencing. qRT-PCR was performed on selected DEGs, including C3, CXCL8, FOS, LTA, S100A8, IL1RL2, NOX1, KCNJ16, FMO1, MT2B and MT1A. Primer sequences were designed using the NCBI primer design tool and synthesized by Qingke Xinye Biotechnology Limited (Changsha, China). Fluorescence quantitative detection was performed using a TIANLONG TL988 fluorescence quantitative PCR instrument (Xi'an Tianlong Technology Limited). Each sample was analyzed in triplicate, and the expression of the target genes was standardized by GAPDH expression. The reaction protocol comprised one cycle of $95^{\circ} \mathrm{C}$ for $1 \mathrm{~min}$ followed by forty cycles of $95^{\circ} \mathrm{C}$ for $10 \mathrm{~s}, 60^{\circ} \mathrm{C}$ for $5 \mathrm{~s}$ and $72^{\circ} \mathrm{C}$ for $10 \mathrm{~s}$. The gene expression was calculated by using the comparative $\mathrm{Ct}\left(2^{-\Delta \Delta \mathrm{Ct}}\right)$ method.

\section{Data availability}

The datasets analyzed during the current study are available in the NCBI Sequence Read Archive (SRA) under BioProject ID: PRJNA 552943.

Received: 12 July 2019; Accepted: 16 October 2019;

Published online: 31 October 2019

\section{References}

1. Rujjanawate, C., Kanjanapothi, D. \& Panthong, A. Pharmacological effect and toxicity of alkaloids from Gelsemium elegans Benth. J Ethnopharmacol 89, 91-95 (2003).

2. Xu, Y. et al. Effects of koumine, an alkaloid of Gelsemium elegans Benth., on inflammatory and neuropathic pain models and possible mechanism with allopregnanolone. Pharmacology, biochemistry, and behavior 101, 504-514, https://doi.org/10.1016/j. pbb.2012.02.009 (2012).

3. Zhou, Z. et al. Poisoning: A Case with 8 Months of Follow-up and Review of the Literature. Front Neurol 8, 204, https://doi. org/10.3389/fneur.2017.00204 (2017).

4. Xie, L. J. \& Han, X. F. Mechanism,clinical characteristics and management of Gelsemium elegans poisoning. Adverse Drug Reactions Journal (2006).

5. Fung, H., Lam, K., Lam, S., Wong, O. \& Kam, C. Two Cases of Gelsemium Elegans Benth. Poisoning. Hong Kong Journal of Emergency Medicine 14, 221-224, https://doi.org/10.1177/102490790701400405 (2007).

6. Xiang, H. et al. Lethal poisoning with Gelsemium elegans in Guizhou, China. Public health 136, 185-187, https://doi.org/10.1016/j. puhe.2016.02.031 (2016)

7. Zhonghua Bencao (Chinese Herbal Medicine). 213-215 (Shanghai Science and Technology Publishing House, 1999).

8. Chen, W., Yang, Y. \& Wu, S. Determination the content of koumine, gelsemine and humantenmine in Fujian Gelsemium elegant. $J$ Fujian Univ TCM 21, 48-50 (2011).

9. Shaffer, C. Next-generation sequencing outpaces expectations. Nature biotechnology 25, 149 (2007).

10. Qi, Y. X., Liu, Y. B. \& Rong, W. H. New transcriptome techniques: RNA-Seq and its application. (Chinese). Genetics 33, 1191-1202 (2011).

11. Anders, S., Pyl, P. T. \& Huber, W. HTSeq-a Python framework to work with high-throughput sequencing data. Bioinformatics 31, 166-169 (2015).

12. Hayes, D. N. \& Kim, W. Y. The next steps in next-gen sequencing of cancer genomes. The Journal of clinical investigation 125, 462-468 (2015)

13. Yang, H.-J., Ratnapriya, R., Cogliati, T., Kim, J.-W. \& Swaroop, A. Vision from next generation sequencing: multi-dimensional genome-wide analysis for producing gene regulatory networks underlying retinal development, aging and disease. Progress in retinal and eye research 46, 1-30 (2015).

14. Dalla-Torre, C. A. et al. Effects of THBS3, SPARC and SPP1 expression on biological behavior and survival in patients with osteosarcoma. BMC cancer 6, 237 (2006).

15. Napolioni, V. et al. Family-based association study of ITGB3 in autism spectrum disorder and its endophenotypes. European journal of human genetics: EJHG 19, 353-359, https://doi.org/10.1038/ejhg.2010.180 (2011).

16. Hu, Y., Shen, F., Crellin, N. K. \& Ouyang, W. The IL-17 pathway as a major therapeutic target in autoimmune diseases. Annals of the New York Academy of Sciences 1217, 60-76 (2011).

17. Harada, A. et al. Essential involvement of interleukin-8 (IL-8) in acute inflammation. Journal of leukocyte biology 56, 559-564 (1994).

18. Huang, G. T., Haake, S. K., Kim, J. W. \& Park, N. H. Differential expression of interleukin-8 and intercellular adhesion molecule-1 by human gingival epithelial cells in response to Actinobacillus actinomycetemcomitans or Porphyromonas gingivalis infection. Oral microbiology and immunology 13, 301-309 (1998).

19. C, C. Pharmaceutials containing diphenhydramine and echinacea and eupatorium and gelsemium and lachesis extracts for the treatment of psorias is and neurodermatitis. Chemical Abstracts 110, 412 (1989).

20. de Sousa, V. D. \& Novel, I. C. pharmacological approaches for the treatment of acne vulgaris. Expert opinion on investigational drugs 23, 1389-1410, https://doi.org/10.1517/13543784.2014.923401 (2014).

21. Lukens, J. R. et al. Dietary modulation of the microbiome affects autoinflammatory disease. Nature 516, 246-249, https://doi. org/10.1038/nature13788 (2014). 
22. Qu, J. et al. Bisindole alkaloids with neural anti-inflammatory activity from Gelsemium elegans. J Nat Prod 76, 2203-2209, https:// doi.org/10.1021/np4005536 (2013).

23. Marzotto, M. et al. Extreme sensitivity of gene expression in human SH-SY5Y neurocytes to ultra-low doses of Gelsemium sempervirens. Bmc Complementary \& Alternative Medicine 14, 104 (2014).

24. Zhang, L. L., Huang, C. Q., Zhang, Z. Y. \& Wang, Z. R. \& Lin J. M. Therapeutic Effect of Gelsemium elegans on Psoriasis-like Animal Model. (Chinese) Journal of the first military medical university, 547-549(2005).

25. Yang, J. et al. Effects of Koumine on Adjuvant- and Collagen-Induced Arthritis in Rats. Journal of Natural Products 79, 2635 (2016).

26. Jin, G. L. et al. The analgesic effect and possible mechanisms by which koumine alters type II collagen-induced arthritis in rats. Journal of Natural Medicines, 1-9 (2018).

27. Yuan, Z. H. et al. Effect of Koumine on LPS-induced RAW264. 7 cell inflammation. (Chinese). Chinese Veterinary Journal 37, $1553-1557$ (2017).

28. Das, L. \& Vinayak, M. Curcumin attenuates carcinogenesis by down regulating proinflammatory cytokine interleukin-1 (IL-1 $\alpha$ and IL-1 $\beta$ ) via modulation of AP-1 and NF-IL6 in lymphoma bearing mice. International immunopharmacology 20, 141-147, https://doi. org/10.1016/j.intimp.2014.02.024 (2014).

29. Dragon, S. et al. IL-17 enhances IL-1beta-mediated CXCL-8 release from human airway smooth muscle cells. American journal of physiology. Lung cellular and molecular physiology 292, L1023-1029, https://doi.org/10.1152/ajplung.00306.2006 (2007).

30. Sun, Y., Guo, Q. M., Liu, D. L., Zhang, M. Z. \& Shu, R. In vivo expression of Toll-like receptor 2, Toll-like receptor 4, CSF2 and LY64 in Chinese chronic periodontitis patients. Oral diseases 16, 343-350, https://doi.org/10.1111/j.1601-0825.2009.01630.x (2010).

31. Singer, L. et al. Inherited complement C3 deficiency: reduced C3 mRNA and protein levels in a Laotian kindred. Clinical immunology and immunopathology 81, 244-252, https://doi.org/10.1006/clin.1996.0185 (1996).

32. Xu, D. et al. Complement C5 Gene Confers Risk for Acute Anterior Uveitis. Investigative ophthalmology \& visual science 56, 4954-4960, https://doi.org/10.1167/iovs.15-16645 (2015).

33. Chimenti, M. S. et al. S100A8/A9 in psoriatic plaques from patients with psoriatic arthritis. The Journal of international medical research 44, 33-37, https://doi.org/10.1177/0300060515598900 (2016).

34. Yang, J. Regulation Mechanism of Koumine on T Helper Cell Polarization Against Rheumatoid Arthritis. (Chinese) Fujian Medical University (2017)

35. Aggarwal, A., Srivastava, R. \& Agrawal, S. T cell responses to citrullinated self-peptides in patients with rheumatoid arthritis. Rheumatology International 33, 2359-2363 (2013).

36. Dobin, A. et al. STAR: ultrafast universal RNA-seq aligner. Bioinformatics 29, 15-21, https://doi.org/10.1093/bioinformatics/bts635 (2013).

37. Franceschini, A. et al. STRING v9. 1: protein-protein interaction networks, with increased coverage and integration. Nucleic acids research 41, D808-D815 (2012).

38. Huang da, W., Sherman, B. T. \& Lempicki, R. A. Bioinformatics enrichment tools: paths toward the comprehensive functional analysis of large gene lists. Nucleic acids research 37, 1-13, https://doi.org/10.1093/nar/gkn923 (2009).

39. Kanehisa, M. \& Goto, S. KEGG: kyoto encyclopedia of genes and genomes. Nucleic acids research 28, 27-30 (2000).

\section{Acknowledgements}

This work was supported by the Hunan Provincial Natural Science Foundation of China (No. 2017JJ1017) and Scientific Research Fund of Hunan Provincial Education Department (No. 18A088).

\section{Author contributions}

K.Y. and Z.Y.L. participated in creating the experimental design; C.Y.H., K.Y., J.J.C., Y.J.L., Z.Y.W. and H.Y. handled animals and collected samples; C.Y.H., X.F.Z., Z.Y.L. and Z.L.S. analyzed the data and discussed the obtained results; C.Y.H. and Z.Y.L. wrote the manuscript; C.Y.H. formatted the figures and tables. All authors read and approved the manuscript.

\section{Competing interests}

The authors declare no competing interests.

\section{Additional information}

Correspondence and requests for materials should be addressed to Z.-Y.L.

Reprints and permissions information is available at www.nature.com/reprints.

Publisher's note Springer Nature remains neutral with regard to jurisdictional claims in published maps and institutional affiliations.

Open Access This article is licensed under a Creative Commons Attribution 4.0 International License, which permits use, sharing, adaptation, distribution and reproduction in any medium or format, as long as you give appropriate credit to the original author(s) and the source, provide a link to the Creative Commons license, and indicate if changes were made. The images or other third party material in this article are included in the article's Creative Commons license, unless indicated otherwise in a credit line to the material. If material is not included in the article's Creative Commons license and your intended use is not permitted by statutory regulation or exceeds the permitted use, you will need to obtain permission directly from the copyright holder. To view a copy of this license, visit http://creativecommons.org/licenses/by/4.0/.

(C) The Author(s) 2019 